\title{
Errorless and errorful learning modulated by transcranial direct current stimulation
}

\author{
Anke Hammer ${ }^{1}$, Bahram Mohammadi ${ }^{1,2}$, Marlen Schmicker ${ }^{3}$, Sina Saliger ${ }^{1}$ and Thomas F Münte ${ }^{1 *}$
}

\begin{abstract}
Background: Errorless learning is advantageous over trial and error learning (errorful learning) as errors are avoided during learning resulting in increased memory performance. Errorful learning challenges the executive control system of memory processes as the erroneous items compete with the correct items during retrieval. The left dorsolateral prefrontal cortex (DLPFC) is a core region involved in this executive control system. Transcranial direct current stimulation (tDCS) can modify the excitability of underlying brain functioning.

Results: In a single blinded tDCS study one group of young healthy participants received anodal and another group cathodal tDCS of the left DLPFC each compared to sham stimulation. Participants had to learn words in an errorless and an errorful manner using a word stem completion paradigm. The results showed that errorless compared to errorful learning had a profound effect on the memory performance in terms of quality. Anodal stimulation of the left DLPFC did not modulate the memory performance following errorless or errorful learning. By contrast, cathodal stimulation hampered memory performance after errorful learning compared to sham, whereas there was no modulation after errorless learning.

Conclusions: Concluding, the study further supports the advantages of errorless learning over errorful learning. Moreover, cathodal stimulation of the left DLPFC hampered memory performance following the conflict-inducing errorful learning as compared to no modulation after errorless learning emphasizing the importance of the left DLPFC in executive control of memory.
\end{abstract}

\section{Background}

The executive control system enables us to learn, to plan behavior, and to inhibit incorrect behavior. With regard to memory functions, often several pieces of associatively linked information (e.g. several names associated to one face) may be available during retrieval and thus incorrect information might interfere with the correct information. Clinically, the inability to distinguish between correctly and incorrectly associated information might lead to neuropsychological conditions such as frontal confabulatory disorder [1]. In experimental work on memory-failures, one important theory to explain false memories, for example the Deese-Roediger-McDermott paradigm [2,3], draws on the notion of falsely activated information [4]. In other words, there appears to be a need to control for and reject falsely associated information during memory retrieval.

\footnotetext{
* Correspondence: thomas.muente@neuro.uni-luebeck.de

'Dept. of Neurology, University of Lübeck, Ratzeburger Allee 160, 23538

Lübeck, Germany

Full list of author information is available at the end of the article
}

One method to study the role of executive control processes during memory retrieval is to modulate the excitability of the prefrontal cortex (PFC). The PFC plays an essential role in the integration of information and the management of multiple tasks [5] as it is crucial in conducing higher cognitive functions, i.e. executive functions including working memory, planning, goal-oriented behaviour, role learning, attention and inhibition and control [e.g. 6, 7-23]. Another method is the use of different encoding modes, i.e. errorful and errorless learning, designed to manipulate the presence of interfering information, which needs to be controlled during memory retrieval [24-26]. Hence, the combination of both, the modulation of the excitability of the PFC and the use of different encoding modes enables us to investigate the importance of executive control processes during memory retrieval depending on the PFC.

As mentioned above, the modulation of the excitability of a specific region may help to clarify the role of this region during a cognitive task. Transcranial direct 
current stimulation (tDCS) is a noninvasive technique for such a modulation as it changes the cortical excitability depending on the polarity of the applied weak current. In general, anodal tDCS increases and cathodal tDCS decreases the neural firing rates, probably due to an induced change in the resting membrane potential [27-29]. These changes lead to corresponding changes in cortical functions: e.g. the excitation of the motor cortex by anodal tDCS leads to increased motor responses and the inhibition by cathodal tDCS leads to decreased motor responses [29] resulting in improved or in inhibited functioning of the motor cortical areas. In relation to more complex functions the same has been found to be true for memory processing [30-34], executive functions such as verbal fluency [35], language processing [36] or decision making [37] in healthy subjects [for a review see 38]. Similar findings have been reported after transcranial magnetic stimulation (TMS), another method to modulate the excitability of the underlying cortical tissue [e.g. 39, 40-43].

With regard to memory processes, the effects of tDCS have been mainly assessed with regard to working memory (WM). Anodal but not cathodal stimulation (1 mA, $10 \mathrm{~min}, 35-\mathrm{cm}^{2}$-sized electrodes) over the left prefrontal cortex resulted in improved performance accuracy on a visual letter WM task [31]. However, Marshall et al. [32] reported slowed reaction time in a visual letter WM task during both anodal and cathodal bilateral stimulation over the DLPFC, suggesting that any kind of electrical stimulation hampers neuronal processes related to response selection and preparation. Ohn et al. [34] showed a time dependent effect of anodal stimulation over the left PFC ( $1 \mathrm{~mA}, 35 \mathrm{~cm}^{2}$-sized electrodes) on WM reporting an increased number of correct responses after 30 minutes of stimulation compared to sham, but earlier measurements or error rates did not reveal any stimulation effects. Boggio et al. [44] reported that continuous tDCS for $20 \mathrm{~min}$ at $2 \mathrm{~mA}$ (but not at $1 \mathrm{~mA}$ ) using the same-sized electrodes improved WM in patients with Parkinson's disease.

Other studies focused on different memory components. Marshall and colleagues [33] investigated consolidation of declarative memories and found that bilateral anodal direct current stimulation at the DLPFC affected declarative memory when applied during sleep. Boggio et al. [45] found that false memories were reduced by $73 \%$ when anodal tDCS is applied to the anterior temporal lobes throughout the encoding and retrieval stages. However, veridical memories remained unchanged after stimulation. Finally, Elmer et al. [46] investigated tDCS effects on short-term learning and subsequent long-term retrieval of auditorily presented verbal material, i.e. wordlist learning with immediate and delayed recall. Cathodal stimulation but not anodal stimulation of the left prefrontal cortex $(1.5 \mathrm{~mA})$ disrupted short-term verbal learning but did not hamper longer lasting consolidation processes that are mainly known to be related to mesial temporal lobe areas. The stimulation of the right prefrontal cortex failed to modulate verbal short-term learning and subsequent longterm retrieval. Further research has evidenced facilitation of learning and memory processes by tDCS application to the PFC $[27,47]$.

In summary one can conclude that tDCS is a noninvasive tool to modulate cognitive processes. However, polarity, intensity, duration and site of stimulation, as well as the size of the electrodes are important parameters in the effects of tDCS on memory processes that are not entirely understood [38].

On the cognitive side, one method to study the role of executive control processes during memory retrieval is the use of different encoding modes, errorful and errorless learning $[24,26,48]$. Errorful learning resembles the typical trial-and-error approach. During learning a number of errors are introduced until the correct response is produced. Exactly these errors are likely to cause interference and false memories/intrusions at retrieval. In contrast, during errorless learning - a managed learning mode only the correct information is introduced and errors are avoided during the learning process reducing the later interference during recall. Errorless learning has been found to lead to profound enhancement of memory retrieval in particular in neuropsychiatric conditions associated to memory deficits such as brain injury [24,49-51], Alzheimer disease [52-54] and schizophrenia [55-58]. A critical review of this literature has been provided by Clare and Jones [48]. Following errorful learning, memory impaired patients may not be able to use the remaining resources of their implicit memory, because they are not able to differentiate between errors made during learning and the correctly learned information [24]. Consequently, such patients benefit from errorless learning compared to errorful learning [24,49-53,55-58], as errors are avoided during the studying phase. Baddeley and Wilson [24] assume that the disadvantage for errorful learning in a word fragment completion task is based on the increased activation level of wrong words of the learning phase which leads to interference. In contrast, this interference is diminished within the errorless modus as only one stimulus was presented during learning. In cases, in which retrieval is based mostly on implicit memory processes, errors are committed because it is not possible to differentiate between concurrent items. Thus, episodic or explicit memory processes are needed to resolve the interference in the errorful modus. This interpretation is supported by electrophysiological studies $[25,26,59]$ focusing on the so called error related negativity (ERN). Contrasting errorful and errorless learning implemented in a word-stem completion task resulted in a learning mode effect in particular for the ERN $[25,26,59]$, which was thought to reflect aspects 
related to the memory decision. The modulation of the ERN amplitude in relation to memory decisions was interpreted as reflecting the activity of an internal monitoring device assessing the activation of the two possible decisions, i.e. the veridicality of retrieved memory traces $[25,26,59]$ or, as an alternative interpretation, variations of the ERN amplitude in errorless and errorful learning might be partially explained by the subjects' perceived likelihood of making an error [25,59]. For both, (decision about the veridicality of retrieved memory traces or the error prevention in different levels of perceived error likelihood) executive control mechanisms of retrieved memory are indispensable.

The ERN is a response-related potential, whose neural source has been consistently found in the posterior medial frontal cortex as shown by brain potential source localization studies [23,60-62] and error-related fMRI activity [63-65] with additional contributions from the lateral prefrontal cortex [7,63,66-69]. The PFC, the target region of the present investigation, is known to be involved in higher executive functions as already described above. It is still unclear whether the left PFC is clearly associated to memory retrieval processes or executive processes such as monitoring as the left PFC was activated for both [18]. Elmer and colleagues [46] modulated the PFC bilaterally based on the HERA hemispheric encoding/retrieval model (HERA) [70] as it states that the left PFC is more involved in episodic memory encoding and the right PFC is more involved in episodic memory retrieval. However, the findings of Elmer et al. suggested a lateralization as a function of the material (i.e. verbal material involving the left language dominant hemisphere) rather than the stage (i.e., encoding or retrieval). As we focus on a verbal task, the targeted region in the present investigation was the left PFC. In order to modulate the activity of the left PFC we used tDCS to examine its role in executive control of memory processes using the two different learning modes, i.e. errorless and errorful learning. The combination of both, changed excitability of left prefrontal regions and the modulation of conflict during memory performance aids us to clarify the specific role of the left PFC of the memory system during executive control of memory processes.

Only a paucity of the tDCS studies to date has explored the modulation of prefrontal areas during explicit memory tasks and to our knowledge, none of the studies modulated conflicting information during encoding. We hypothesised in view of the findings of some neuroimaging studies [63-65] and electrophysiological studies on errorless and errorful learning $[25,26,59]$ that verbal memory retrieval should be modulated by the stimulation of the prefrontal cortex, and more specifically the left PFC. In case the left PFC is solely involved in memory processes, we expected a better learning performance following anodal stimulation of the left PFC [31,33,34], and we assumed a decrease in performance during cathodal stimulation [46] independent of the learning mode. However, in case the left PFC plays an important role in executive control during memory processes we expected a different modulation following tDCS driven by learning mode as errorful learning compared to errorless learning enhances conflict processing during retrieval as added errors during learning are likely to cause interference at retrieval.

\section{Results}

Performance measures are shown in Figure 1. Following anodal real or sham stimulation, the errorless learning led to an improved memory performance as compared to the errorful learning mode. Errorless learning was not modulated by anodal or cathodal stimulation as real and sham stimulation resulted in similar discrimination indices. Differences can be observed for errorful learning. Following real cathodal stimulation compared to sham, memory performance after errorful learning was reduced (errorful: dprime stimulation $<$ sham) and vice versa for the anodal stimulation (errorful: dprime stimulation > sham).

The ANOVA crossing the between factor Group (anodal vs. cathodal) and the within factors Stimulation (active vs. sham) and Learning Mode (errorful vs. errorless learning) on the dprime measures revealed a group effect (Group: $\mathrm{F}_{1,34}=4.59, \mathrm{p}<.04$ ), a significant main effect for learning mode (learning mode: $\mathrm{F}_{1,34}=136.61$,
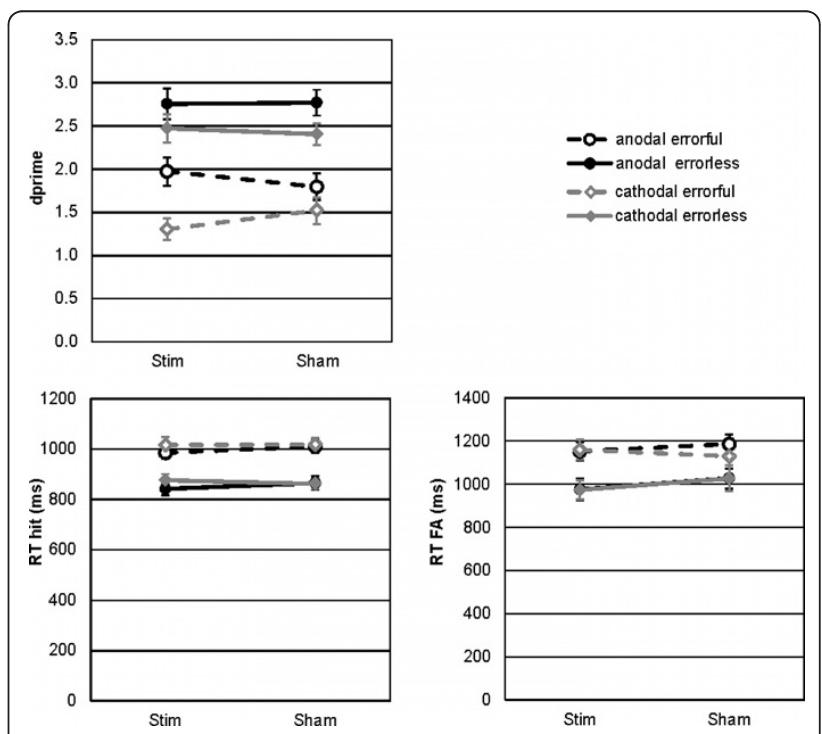

Figure 1 Behavioral data. The plots show the memory performance and the reaction times for EL (solid line) and EF (dashed line) learning after anodal (black), cathodal (grey) or sham stimulation. 
$\mathrm{p}<.001$ ) and a significant triple interaction (Group $\times$ Stimulation $\times$ Learning Mode: $\left.\mathrm{F}_{1,34}=4.68, \mathrm{p}<.04\right)$. The other main effects or interactions failed to reach significance (Stimulation: $\mathrm{F}_{1,34}=0.04, \mathrm{p}>.9$; Stimulation $\times$ Group: $\mathrm{F}_{1,34}=2.35, \mathrm{p}>0.1$, Learning Mode $\times$ Group: $\mathrm{F}_{1,34}=0.81, \mathrm{p}>0.3$, Stimulation $\times$ Learning Mode: $\left.\mathrm{F}_{1,34}=0.17, \mathrm{p}>.6\right)$.

The learning mode effect was statistically significant for all paired comparisons of errorless $>$ errorful (anodal stimulation: $\mathrm{t}_{17}=4.17, \mathrm{p}<.002$; anodal sham: $\mathrm{t}_{17}=6.25$, $\mathrm{p}<.001$; cathodal stimulation: $\mathrm{t}_{17}=14.23, \mathrm{p}<.001$; cathodal sham: $\left.\mathrm{t}_{17}=8.35, \mathrm{p}<.001\right)$. The anodal stimulation effect directly comparing stimulation $>$ sham failed to reach significance for both learning modes (errorful: $t_{17}=$ 1.59, $\mathrm{p}>.1$; errorless: $\mathrm{t}_{17}=-0.13, \mathrm{p}>.9$ ). The cathodal stimulation effect with stimulation $<$ sham was significant for errorful learning $\left(t_{17}=2.35, \mathrm{p}<.04\right)$ but failed to reach significance for errorless learning $\left(t_{17}=-0.61, p>\right.$ $.5)$. As equality between the baseline conditions was given (see method section) we directly compared both groups with independent t-tests. Differences were solely found for the errorful learning mode (cathodal < anodal: $\left.\mathrm{t}_{34}=3.28, \mathrm{p}<.003\right)$ but not errorless learning mode (cathodal $>$ anodal: $\mathrm{t}_{34}=-1.16, \mathrm{p}>.3$ ).

The mean reaction times for hits and false alarms are shown in Figure 1. The repeated measures ANOVA crossing the between factor Group (anodal vs. cathodal) and the within factors Stimulation (active vs. sham) and Learning Mode (errorful vs. errorless learning) on the reaction times for hits indicated significantly faster responses for errorless than for errorful items (mean RT errorless: 861 ms, errorful: $1008 \mathrm{~ms}$; learning mode: $\mathrm{F}_{1,34}=209.94, \mathrm{p}<$ .001). All other main effects or interactions failed to reach significance (Hits: Group $\mathrm{F}_{1,34}=0.28, \mathrm{p}>.5$; Stimulation $\mathrm{F}_{1,34}=0.80, \mathrm{p}>.3$; Stimulation $\times$ Group $\mathrm{F}_{1,34}=2.18, \mathrm{p}>$ .1; LM $\times$ Goup $\mathrm{F}_{1,34}=0.03, \mathrm{p}>.8$; Stimulation $\times \mathrm{LM}$ $\mathrm{F}_{1,34}=0.26, \mathrm{p}>.6$ and Stimulation $\times \mathrm{LM} \times$ Group $\mathrm{F}_{1,34}=$ $0.13, \mathrm{p}>.7)$. The same pattern was observed for false alarms (mean RT errorless: $1001 \mathrm{~ms}$, errorful: $1156 \mathrm{~ms}$; learning mode: $\mathrm{F}_{1,34}=38.86, \mathrm{p}<.001$ ) and no further significant effect (Group $\mathrm{F}_{1,34}=0.28, \mathrm{p}>$.6; Stimulation $\mathrm{F}_{1,34}$ $=0.80, \mathrm{p}>.3$; Stimulation $\times$ Group $\mathrm{F}_{1,34}=2.18, \mathrm{p}>.1$; $\mathrm{LM} \times$ Goup $\mathrm{F}_{1,34}=0.03, \mathrm{p}>.8$; Stimulation $\times \mathrm{LM} \mathrm{F}_{1,34}=$ $0.26, \mathrm{p}>.6$ and Stimulation $\times \mathrm{LM} \times$ Group $\mathrm{F}_{1,34}=0.13$, $\mathrm{p}>.7)$.

\section{Discussion}

The aim of the study was to clarify the role of the left PFC in executive control processes during memory retrieval by comparing two different learning modes (errorless and errorful learning) that tax executive processing to a different degree and anodal and cathodal tDCS to modulate the excitability of the left PFC. Memory processing was driven by learning mode as errorless learning was advantageous over errorful learning. More importantly, cathodal stimulation hampered encoding and memory retrieval after errorful learning but not errorless learning, whereas anodal stimulation did not alter encoding and memory retrieval after errorful or errorless learning.

Errorless learning compared to errorful learning had a profound effect on the memory performance. Additionally, the responses to errorless learned items were faster than to errorful items. These findings underscore the benefits of errorless learning as shown before [24,25,59,71,72]. Following Baddeley and Wilson [24], errorful learning results in increased memory errors due to the enhanced activation of the previously incorrectly guessed items. These will lead to an enhanced conflict during recognition as the activation of the target word and the incorrect guessed words compete with each other. By contrast, errorless learning - as a rather managed learning mode with the aim to avoid errors during learning - has the advantage of less possible distractors that might interfere in later retrieval and thus there is reduced conflict during retrieval. For the given design, one could argue that the active task to generate a sentence with the errorless learned word might be the sole explanation of the errorless learning advantage. However, Rodriguez-Fornells et al. [26] and Heldmann et al. [25] argued that the errorful learning required an additional activity by the participant (i.e. the generation of the candidate words), whereas errorless learning did not, which leads to a different level of processing. In order to equate the level of processing as close as possible we followed the proposal of Heldmann and colleagues [25] to introduce the additional task of sentence generation.

There was no effect of tDCS of the left PFC on encoding and memory performance following errorless learning. Encoding and memory performance after errorful learning was reduced by cathodal as compared to sham stimulation, whereas anodal stimulation did not alter encoding and memory performance after errorful learning.

Anodal stimulation failed to improve memory retrieval irrespective of learning mode in the present investigation, which is different from earlier studies. This might be related to diverse stimulation methods (e.g. position of the reference electrode, stimulation site, type of current, current intensity and duration of stimulation) as pointed out in a thorough review [38]. Additionally, most of the reported positive memory effects after anodal stimulation concern working memory processes $[31,34,44,73]$ and not verbal memory processing. Elmer and colleagues [46] used an auditory word list learning paradigm and did not find a modification of short term learning after unilateral anodal stimulation of the DLPFC compared to sham, which is in accordance with the present finding. Moreover, after cathodal stimulation of the DLPFC Elmer et al. [46] found a decrease of short term verbal learning, which again is in 
accordance with the decrease of memory performance after errorful learning in the present study. The conforming results might be based on the similarities of both studies: the participants had to learn auditorily presented words and had to recall these later on while the left DLPFC was modulated with anodal or cathodal stimulation. However, both studies differed as well. First of all, we did not stimulate the right DLPFC and we used different stimulation settings (Elmer et al. used an enlarged reference electrode and stimulated with a slightly increased direct current of $1.5 \mathrm{~mA}$ instead of $1 \mathrm{~mA}$ and a shorter duration with 5 minutes compared to 30 minutes). Additionally, the paradigm was different. While Elmer's participants listened to and repeated the word list three times, our participants either heard the first three letters and the words once and produced a sentence with the word (errorless learning) or heard the first three letters and had to guess the words once, which introduced errors during the learning (errorful learning).

Why did we not find a decrease of memory performance for the errorless condition? As pointed out above the retrieval after errorless learning is supposed to be rather conflict free as errors are avoided during learning and thus, nearly no conflicting memory traces are present in later recognition. This greatly reduces the processing demands for the PFC and thus no effect of stimulation can be seen. By contrast, errorful learning challenges the executive control system as erroneous items from the learning phase compete with the correct items during recognition. We hypothesized that cathodal stimulation reduced executive control of memory resulting in a reduced memory performance. These results dovetail nicely with the Activation-Monitoring framework [74] developed to explain false memories. This framework emphasizes that during retrieval one has to differentiate between highly activated but non-presented critical words and studied words to avoid false memories. By this account false memories, for example in the Deese-Roediger-McDermott paradigm, are due to a failure in monitoring processes differentiating falsely from correctly activated words. In a similar vein, greater activations of the dorsolateral prefrontal cortex during false than true recognition have been interpreted as reflecting monitoring-processes induced by the strong sense of familiarity associated with false memories [e.g. 75]. Differentially spoken, disrupting the processing of the left DLPFC via cathodal tDCS disturbs monitoring processes during memory retrieval and hampers the differentiation between correctly and erroneously words learned during errorful learning.

\section{Limitations}

We would like to mention methodological limitations of the present tDCS protocol. The reference electrode placed in the contralateral supraorbital region (either the anode or the cathode) with the same size of the active electrode can also induce reference specific effects (ano$\mathrm{dal} /$ cathodal) in parallel to the cathodal/anodal effects of the active electrode. However, the present settings were used in well-established tDCS protocols to modulate the left DLPFC [31,34,76,77]. Another limitation in terms of the stimulation protocol is that the four learning phases and four recognition phases were done in an alternating fashion. We therefore cannot differentiate between the stimulation influences on encoding or retrieval. However, the given protocol appeared to offer the best trade-off between a sufficient number of stimuli and a viable task for participants.

\section{Conclusions}

Altogether we conclude that cathodal tDCS hampered executive control mechanisms during encoding and verbal memory processes and led to effects when retrieval conflict was induced by the errorful learning mode. This underscores the role of the left PFC in the control of encoding and verbal memory retrieval. On a more general level, the present study attests to the potential of the tDCS to study the neural substrates of cognitive functions.

\section{Methods}

\section{Participants}

Forty healthy participants were split into two groups. Four datasets had to be removed due to technical problems with the button responses. The first group $(\mathrm{N}=18$; age $23.3+/$ - 3.0 years; 13 women) received both anodal and sham tDCS over the left prefrontal cortex. The second group $(\mathrm{N}=18$; age $23.0+/-3.4$ years; 13 women) received both cathodal and sham tDCS over the left prefrontal cortex. All participants were right-handed, and had no history of neuropsychiatric or cardiovascular disease. Written informed consent was obtained from all participants before they entered the study, and the study protocol was approved by the local ethics committee of Magdeburg.

\section{Experimental protocol}

This study was designed as a single-blind, crossover, sham controlled experiment. All participants participated in both active and sham tDCS. The order of stimulation was counterbalanced and randomized across all participants. To minimize carryover effects, the interval between sessions was seven days minimum. Initially, the participants were familiarized with the cognitive tasks.

\section{Cognitive paradigm}

To evaluate changes in the cognitive control system of memory performance after tDCS, we used a recognition memory task requiring a yes/no response to items that had been acquired during errorless and errorful learning 
[nearly identical to 25,26 ]. Subjects participated in one errorful learning block and one errorless learning block per session (i.e. stimulation or sham session). The order of learning blocks was counterbalanced across subjects. One block comprised two runs each composed of a learning phase and a subsequent recognition phase resulting in two errorless and two errorful runs per session. In each run each participant performed a wordfragment-completion task for 30 word-fragments [identical to 25,26$]$. In the errorful condition, the first three letters of a word were given by the experimenter and the subject was asked to guess words to complete this fragment. After guessing, the experimenter revealed which word was the target word to be remembered. If subjects failed to guess the intended target word, the experimenter introduced example words and the target word. For each of the presented word-fragments at least two German words exist with a high and comparable guessing probability, e.g. ANZ: 'Anzeige' [advertisement], 'Anzahl' [number] $[25,26]$. The assignment of the target candidate for each word-fragment was balanced across subjects. There were four different assignments (see Table 1) which were systematically rotated over participants.

For each fragment one word was used during the learning phase as a target word, while the other high frequency alternative was used as distracter during the recognition phase. In the errorless learning condition the first three letters of the word were introduced by the experimenter directly followed by the target word. During errorful learning the participants guessed several words to complete the word-fragment which resulted in deeper processing of words as compared to errorless word list learning. To ensure such a deeper processing of words in the errorless condition as well, participants had to produce a sentence with the word. During each recognition phase 30 targets, 30 distracters and 30 additional new words were presented in a randomized order. There were two errorless and two errorful runs per session (active or sham tDC-stimulation) resulting in 120 words per stimulus type per session.

The task was to indicate by button press (right index/ middle finger), whether or not a given word was a target word. The participants did not receive feedback about

Table 1 Example for an assignment of candidate words to the learning lists

\begin{tabular}{lllll}
\hline Condition & List A & List B & List C & List D \\
\hline Errorless target & Hafen & Hafer & Anzeige & Anzahl \\
Errorless non-target & Hafer & Hafen & Anzahl & Anzeige \\
Errorful target & Anzeige & Anzahl & Hafen & Hafer \\
Errorful nontarget & Anzahl & Anzeige & Hafer & Hafen \\
New word & Feder & Feder & Feder & Feder \\
\hline
\end{tabular}

Translations: Hafen - habor, Hafer - oat, Anzeige - advertisement, Anzahlnumber, Feder - feather. the correctness of the actual response. The words were presented in white letters on a black background in the middle of a computer screen. Stimuli subtended $0.57^{\circ}$ in height and between $1.7^{\circ}$ and $4.9^{\circ}$ in width. The stimulus duration was $300 \mathrm{~ms}$ with a stimulus-onset-asynchrony between 1800 and 2500 ms. Altogether, each run lasted 15 minutes including the learning and recognition phase.

\section{Transcranial direct current stimulation application}

Direct current was transferred by a saline-soaked pair of surface sponge electrodes $\left(35 \mathrm{~cm}^{2}\right)$ and delivered by a specially developed, battery-driven, constant current stimulator (eldith, neuroConn GmbH, Ilmenau, Germany). For anodal stimulation of the left dorsolateral prefrontal cortex, the anode was placed over position F3 (according to the 10-20 international system for electroencephalogram electrode placement), and the cathode was placed over the contralateral right supraorbital area [31,34,76,77]. For cathodal stimulation of the left dorsolateral prefrontal cortex, the cathode was placed over F3 and the anode over the contralateral right supraorbital area. A constant current of $1 \mathrm{~mA}$ was applied for 30 min starting 10 minutes before the first learning phase. This timing was motivated by the results of Ohn and colleagues [34] who reported an increase of WM accuracy after 20 minutes of anodal stimulation compared to baseline and increased accuracy after 30 minutes of anodal stimulation compared to baseline and sham stimulation with the same stimulation settings $\left(35 \mathrm{~cm}^{2}\right.$ electrodes, $1 \mathrm{~mA}$, anode was placed at F3 position, reference placed at the contralateral right supraorbital area). These effects diminished after an hour of anodal stimulation. Here, the first recognition phase started around 20 minutes after stimulation onset (10 minutes waiting and around 10 minutes learning phase) and the fourth and last recognition phase started at around 65 minutes after stimulation onset. With the present timing of stimulation we sought to tap into the maximal tDCS effects similar to Ohn et al. [34]. After the stimulator had been turned off, the electrodes were kept in place until the end of measurement. For sham stimulation, the same electrode placements were used, but the current was applied for $8 \mathrm{~s}$ and was then turned off. This procedure ensured the blinding of the subjects for real or sham stimulation as they also felt the initial itching sensation for the first seconds of tDCS [31,38].

\section{Data analysis}

The primary outcomes of this study were the response times (hits and false alarms) and signal detection measure d' (accuracy). Here we focus on the discrimination index (d') as it describes the subject's ability to discriminate between old and new items in memory recognition [78]. Values for d' were estimated by the $\mathrm{z}$-score of the false alarm rate minus the $\mathrm{z}$-score of the hit rate. 
T-tests between the baseline conditions (i.e. sham stimulation) of both groups were calculated in order to evaluate the equality of both groups in baseline performance: The comparisons of the performances of both groups following sham stimulation did not reveal any significant differences in baseline performances between the two groups (errorless: $\mathrm{t}_{34}=-1.38, \mathrm{p}>.1$; errorful: $\left.\mathrm{t}_{34}=-1.26, \mathrm{p}>.2\right)$. The reaction times for hits (errorless: $\mathrm{t}_{34}=0.83, \mathrm{p}>.9$; errorful: $\mathrm{t}_{34}=-0.19, \mathrm{p}>.8$ ) and for False Alarms (errorless: $\mathrm{t}_{34}=$ $0.02, p>.9$; errorful: $\mathrm{t}_{34}=0.90, \mathrm{p}>.3$ ) following sham stimulations were not significantly different between both groups.

Measures were subjected into a repeated measures ANOVA including the between factor Group (anodal vs. cathodal) and the within factors Stimulation (active vs. sham) and Learning Mode (errorful vs. errorless learning). Differences were further analyzed by paired t-tests within groups and by independent t-tests between groups.

\section{Acknowledgements}

The work was supported by grants from the DFG and the BMBF to TFM.

\section{Author details}

'Dept. of Neurology, University of Lübeck, Ratzeburger Allee 160, 23538

Lübeck, Germany. ${ }^{2}$ Neurology, International Neuroscience Institute (INI), Rudolf-Pichlmayr-Straße 4, 30625, Hannover, Germany. ${ }^{3}$ Institute of Medical Psychology, Otto-von-Guericke University, Leipziger Straße 44, 39120 Magdeburg, Germany.

\section{Authors' contributions}

$\mathrm{AH}$ conceived and designed the experiments, performed the statistical analysis and drafted the manuscript. BM contributed to the design and revised it critically for important intellectual content. MS performed the experiments, contributed to the statistical analysis and revised the manuscript critically for important intellectual content. SS performed the experiments, contributed to the statistical analysis and revised the manuscript critically for important intellectual content. TFM conceived and designed the experiment and drafted the manuscript. All authors read and approved the final manuscript.

Received: 9 May 2011 Accepted: 22 July 2011 Published: 22 July 2011

\section{References}

1. Burgess PW, Shallice T: Confabulation and the control of recollection. Memory 1996, 4(4):359-411.

2. Deese J: On the prediction of occurrence of particular verbal intrusions in immediate recall. J Exp Psychol 1959, 58(1):17-22.

3. Roediger HL, McDermott KB: Creating False Memories: Remembering Words not Presented in Lists. Journal of Experimental Psychology: Learning, Memory, and Cognition 1995, 21(4):803-814.

4. Brainerd CJ, Reyna VF: Fuzzy-trace theory: dual processes in memory, reasoning, and cognitive neuroscience. Adv Child Dev Behav 2001, 28:41-100

5. Reynolds JR, McDermott KB, Braver TS: A direct comparison of anterior prefrontal cortex involvement in episodic retrieval and integration. Cereb Cortex 2006, 16(4):519-528.

6. Dobbins IG, Foley H, Schacter DL, Wagner AD: Executive control during episodic retrieval: multiple prefrontal processes subserve source memory. Neuron 2002, 35(5):989-996.

7. Fassbender C, Murphy K, Foxe JJ, Wylie GR, Javitt DC, Robertson $\|_{\text {, }}$ Garavan $\mathrm{H}$ : A topography of executive functions and their interactions revealed by functional magnetic resonance imaging. Brain Res Cogn Brain Res 2004, 20(2):132-143.
8. Faw B: Pre-frontal executive committee for perception, working memory, attention, long-term memory, motor control, and thinking: a tutorial review. Conscious Cogn 2003, 12(1):83-139.

9. Fletcher $\mathrm{PC}$, Shallice T, Dolan RJ: The functional roles of prefrontal cortex in episodic memory. I Encoding Brain 1998, 121(Pt 7):1239-1248.

10. Kim H, Cabeza R: Common and specific brain regions in high- versus low-confidence recognition memory. Brain Res 2009, 1282:103-113.

11. Koechlin E, Ody C, Kouneiher F: The architecture of cognitive control in the human prefrontal cortex. Science 2003, 302(5648):1181-1185.

12. Kondo H, Morishita M, Osaka N, Osaka M, Fukuyama H, Shibasaki H: Functional roles of the cingulo-frontal network in performance on working memory. Neuroimage 2004, 21(1):2-14.

13. Markela-Lerenc J, Ille N, Kaiser S, Fiedler P, Mundt C, Weisbrod M: Prefrontal-cingulate activation during executive control: which comes first? Brain Res Cogn Brain Res 2004, 18(3):278-287.

14. Poppenk J, Moscovitch M, McIntosh AR, Ozcelik E, Craik Fl: Encoding the future: successful processing of intentions engages predictive brain networks. Neuroimage 2010, 49(1):905-913.

15. Postle BR, Brush LN, Nick AM: Prefrontal cortex and the mediation of proactive interference in working memory. Cogn Affect Behav Neurosci 2004, 4(4):600-608.

16. Race EA, Shanker $S$, Wagner AD: Neural priming in human frontal cortex: multiple forms of learning reduce demands on the prefrontal executive system. J Cogn Neurosci 2009, 21(9):1766-1781.

17. Rajah MN, Ames B, D'Esposito M: Prefrontal contributions to domaingeneral executive control processes during temporal context retrieval. Neuropsychologia 2008, 46(4):1088-1103.

18. Rajah MN, Mclntosh AR: Dissociating prefrontal contributions during a recency memory task. Neuropsychologia 2006, 44(3):350-364.

19. Raye $\mathrm{CL}$, Johnson MK, Mitchell KJ, Greene EJ, Johnson MR: Refreshing: a minimal executive function. Cortex 2007, 43(1):135-145.

20. Rypma B, Berger JS, Prabhakaran V, Bly BM, Kimberg DY, Biswal BB, D'Esposito M: Neural correlates of cognitive efficiency. Neuroimage 2006, 33(3):969-979.

21. Taylor SF, Welsh RC, Wager TD, Phan KL, Fitzgerald KD, Gehring WJ: A functional neuroimaging study of motivation and executive function Neuroimage 2004, 21(3):1045-1054.

22. Wagner AD, Maril A, Bjork RA, Schacter DL: Prefrontal contributions to executive control: $\mathrm{fMRI}$ evidence for functional distinctions within lateral Prefrontal cortex. Neuroimage 2001, 14(6):1337-1347.

23. Herrmann M, Rotte $M$, Grubich $C$, Ebert AD, Schiltz K, Munte TF, Heinze HJ: Control of semantic interference in episodic memory retrieval is associated with an anterior cingulate-prefrontal activation pattern. Hum Brain Mapp 2001, 13(2):94-103.

24. Baddeley A, Wilson BA: When implicit learning fails: amnesia and the problem of error elimination. Neuropsychologia 1994, 32(1):53-68.

25. Heldmann M, Markgraf U, Rodriguez-Fornells A, Munte TF: Brain potentials reveal the role of conflict in human errorful and errorless learning. Neurosci Lett 2008.

26. Rodriguez-Fornells A, Kofidis C, Munte TF: An electrophysiological study of errorless learning. Brain Res Cogn Brain Res 2004, 19(2):160-173.

27. Floel A, Cohen LG: Contribution of noninvasive cortical stimulation to the study of memory functions. Brain Research Reviews 2007, 53(2):250-259.

28. Lang N, Siebner HR, Ward NS, Lee L, Nitsche MA, Paulus W, Rothwell JC, Lemon RN, Frackowiak RS: How does transcranial DC stimulation of the primary motor cortex alter regional neuronal activity in the human brain? Eur J Neurosci 2005, 22(2):495-504.

29. Nitsche MA, Paulus W: Excitability changes induced in the human motor cortex by weak transcranial direct current stimulation. J Physio/ 2000, 527(Pt 3):633-639.

30. Ferrucci $R$, Marceglia S, Vergari M, Cogiamanian F, Mrakic-Sposta S, Mameli F, Zago S, Barbieri S, Priori A: Cerebellar transcranial direct current stimulation impairs the practice-dependent proficiency increase in working memory. J Cogn Neurosci 2008, 20(9):1687-1697.

31. Fregni F, Boggio PS, Nitsche M, Bermpohl F, Antal A, Feredoes $E$, Marcolin MA, Rigonatti SP, Silva MT, Paulus W, et al: Anodal transcranial direct current stimulation of prefrontal cortex enhances working memory. Exp Brain Res 2005, 166(1):23-30.

32. Marshall L, M"lle M, Siebner HR, Born J: Bifrontal transcranial direct current stimulation slows reaction time in a working memory task. $B M C$ Neuroscience 2005, 6. 
33. Marshall L, Molle M, Hallschmid M, Born J: Transcranial direct current stimulation during sleep improves declarative memory. J Neurosci 2004, 24(44):9985-9992.

34. Ohn SH, Park Cl, Yoo WK, Ko MH, Choi KP, Kim GM, Lee YT, Kim YH: Timedependent effect of transcranial direct current stimulation on the enhancement of working memory. NeuroReport 2008, 19(1):43-47.

35. Iyer MB, Mattu U, Grafman J, Lomarev M, Sato S, Wassermann EM: Safety and cognitive effect of frontal DC brain polarization in healthy individuals. Neurology 2005, 64(5):872-875.

36. Sparing R, Dafotakis M, Meister IG, Thirugnanasambandam N, Fink GR: Enhancing language performance with non-invasive brain stimulation-A transcranial direct current stimulation study in healthy humans. Neuropsychologia 2008, 46(1):261-268.

37. Fecteau S, Pascual-Leone A, Zald DH, Liguori P, Theoret H, Boggio PS, Fregni F: Activation of prefrontal cortex by transcranial direct current stimulation reduces appetite for risk during ambiguous decision making. J Neurosci 2007, 27(23):6212-6218.

38. Nitsche MA, Cohen LG, Wassermann EM, Priori A, Lang N, Antal A, Paulus W, Hummel F, Boggio PS, Fregni F, et al: Transcranial direct current stimulation: State of the art 2008. Brain Stimulation 2008, 1(3):206-223.

39. Basso D, Ferrari M, Palladino P: Prospective memory and working memory: asymmetrical effects during frontal lobe TMS stimulation. Neuropsychologia 2010, 48(11):3282-3290.

40. Gagnon G, Blanchet S, Grondin S, Schneider C: Paired-pulse transcranial magnetic stimulation over the dorsolateral prefrontal cortex interferes with episodic encoding and retrieval for both verbal and non-verbal materials. Brain Res 2010, 1344:148-158.

41. Innocenti I, Giovannelli F, Cincotta M, Feurra M, Polizzotto NR, Bianco G, Cappa SF, Rossi S, Basso D, Ferrari M, et al: Event-related rTMS at encoding affects differently deep and shallow memory traces. Neuroimage 2010, 53(1):325-330.

42. Rami L, Gironell A, Kulisevsky J, Garcia-Sanchez C, Berthier M, EstevezGonzalez A: Effects of repetitive transcranial magnetic stimulation on memory subtypes: a controlled study. Neuropsychologia 2003, 41(14):1877-1883.

43. Reis J, Robertson E, Krakauer JW, Rothwell J, Marshall L, Gerloff C, Wassermann E, Pascual-Leone A, Hummel F, Celnik PA, et al: Consensus: "Can tDCS and TMS enhance motor learning and memory formation?". Brain Stimulat 2008, 1(4):363-369.

44. Boggio PS, Ferrucci R, Rigonatti SP, Covre P, Nitsche M, Pascual-Leone A, Fregni F: Effects of transcranial direct current stimulation on working memory in patients with Parkinson's disease. J Neurol Sci 2006, 249(1):31-38.

45. Boggio PS, Fregni F, Valasek C, Ellwood S, Chi R, Gallate J, Pascual-Leone A, Snyder A: Temporal lobe cortical electrical stimulation during the encoding and retrieval phase reduces false memories. PLoS One 2009, 4(3):e4959.

46. Elmer S, Burkard M, Renz B, Meyer M, Jancke L: Direct current induced short-term modulation of the left dorsolateral prefrontal cortex while learning auditory presented nouns. Behav Brain Funct 2009, 5:29.

47. Kincses TZ, Antal A, Nitsche MA, Bartfai O, Paulus W: Facilitation of probabilistic classification learning by transcranial direct current stimulation of the prefrontal cortex in the human. Neuropsychologia 2004, 42(1):113-117.

48. Clare L, Jones RS: Errorless Learning in the Rehabilitation of Memory Impairment: A Critical Review. Neuropsychol Rev 2008.

49. Glisky EL, Schacter DL, Tulving E: Learning and retention of computerrelated vocabulary in memory-impaired patients: method of vanishing cues. J Clin Exp Neuropsychol 1986, 8(3):292-312.

50. Hunkin NM, Squires EJ, Parkin AJ, Tidy JA: Are the benefits of errorless learning dependent on implicit memory? Neuropsychologia 1998, 36(1):25-36.

51. Squires EJ, Hunkin NM, Parkin AJ: Errorless learning of novel associations in amnesia. Neuropsychologia 1997, 35(8):1103-1111.

52. Clare L, Wilson BA, Carter G, Breen K, Gosses A, Hodges JR: Intervening with everyday memory problems in dementia of Alzheimer type: an errorless learning approach. J Clin Exp Neuropsychol 2000, 22(1):132-146.

53. Clare L, Wilson BA, Carter G, Hodges JR: Cognitive rehabilitation as a component of early intervention in Alzheimer's disease: a single case study. Aging Ment Health 2003, 7(1):15-21.
54. Clare L, Wilson BA, Carter G, Roth I, Hodges JR: Relearning face-name associations in early Alzheimer's disease. Neuropsychology 2002, 16(4):538-547

55. Kern RS, Green MF, Mitchell S, Kopelowicz A, Mintz J, Liberman RP: Extensions of errorless learning for social problem-solving deficits in schizophrenia. Am J Psychiatry 2005, 162(3):513-519.

56. Kern RS, Liberman RP, Kopelowicz A, Mintz J, Green MF: Applications of errorless learning for improving work performance in persons with schizophrenia. Am J Psychiatry 2002, 159(11):1921-1926.

57. O'Carroll RE, Russell HH, Lawrie SM, Johnstone EC: Errorless learning and the cognitive rehabilitation of memory-impaired schizophrenic patients. Psychol Med 1999, 29(1):105-112.

58. Pope JW, Kern RS: An "errorful" learning deficit in schizophrenia? J Clin Exp Neuropsychol 2006, 28(1):101-110.

59. Hammer A, Kordon A, Heldmann M, Zurowski B, Munte TF: Brain potentials of conflict and error-likelihood following errorful and errorless learning in obsessive-compulsive disorder. PLoS One 2009, 4(8):e6553.

60. Dehaene S, Posner MI, Tucker DM: Localization of a neural system for error detection and compensation. Psychological Science 1994, 5(5):303-305.

61. Luu P, Tucker DM: Regulating action: alternating activation of midline frontal and motor cortical networks. Clinical Neurophysiology 2001, 112(7):1295-1306.

62. van Veen V, Holroyd CB, Cohen JD, Stenger VA, Carter CS: Errors without conflict: implications for performance monitoring theories of anterior cingulate cortex. Brain Cogn 2004, 56(2):267-276.

63. Ridderinkhof KR, Ullsperger $M$, Crone EA, Nieuwenhuis S: The role of the medial frontal cortex in cognitive control. Science 2004, 306(5695):443-447

64. Ullsperger M, von Cramon DY: Subprocesses of performance monitoring: a dissociation of error processing and response competition revealed by event-related fMRI and ERPs. Neuroimage 2001, 14(6):1387-1401.

65. Ullsperger M, von Cramon DY: Decision making, performance and outcome monitoring in frontal cortical areas. Nat Neurosci 2004, 7(11):1173-1174.

66. van Veen V, Carter CS: The anterior cingulate as a conflict monitor: fMRI and ERP studies. Physiol Behav 2002, 77(4-5):477-482.

67. Gehring WJ, Knight RT: Prefrontal-cingulate interactions in action monitoring. Nat Neurosci 2000, 3(5):516-520.

68. Marco-Pallares J, Camara E, Munte TF, Rodriguez-Fornells A: Neural mechanisms underlying adaptive actions after slips. J Cogn Neurosci 2008, 20(9):1595-1610.

69. van Veen V, Cohen JD, Botvinick MM, Stenger VA, Carter CS: Anterior cingulate cortex, conflict monitoring, and levels of processing. Neuroimage 2001, 14(6):1302-1308.

70. Habib R, Nyberg L, Tulving E: Hemispheric asymmetries of memory: the HERA model revisited. Trends Cogn Sci 2003, 7(6):241-245.

71. Kessels RP, Boekhorst ST, Postma A: The contribution of implicit and explicit memory to the effects of errorless learning: a comparison between young and older adults. J Int Neuropsychol Soc 2005, 11(2):144-151.

72. Kessels RP, de Haan EH: Mnemonic strategies in older people: a comparison of errorless and errorful learning. Age Ageing 2003, 32(5):529-533.

73. Zaehle T, Sandmann P, Thorne JD, Jancke L, Herrmann CS: Transcranial direct current stimulation of the prefrontal cortex modulates working memory performance: combined behavioural and electrophysiological evidence. BMC Neurosci 2011, 12:2.

74. Balota DA, Cortese MJ, Duchek JM, Adams D, Roediger HL, McDermott KB, Yerys BM: Veridical and false memories in healthy older adults and in dementia of the Alzheimer's type. Cogn Neuropsychol 1999, 16(35):361-384.

75. Schacter DL, Reiman E, Curran T, Yun LS, Bandy D, McDermott KB, Roediger HL: Neuroanatomical correlates of veridical and illusory recognition memory: evidence from positron emission tomography. Neuron 1996, 17(2):267-274.

76. Fregni $F$, Boggio PS, Santos MC, Lima M, Vieira AL, Rigonatti SP, Silva MT, Barbosa ER, Nitsche MA, Pascual-Leone A: Noninvasive cortical stimulation with transcranial direct current stimulation in Parkinson's disease. Mov Disord 2006, 21(10):1693-1702. 
77. Siebner HR, Lang N, Rizzo V, Nitsche MA, Paulus W, Lemon RN, Rothwell JC: Preconditioning of low-frequency repetitive transcranial magnetic stimulation with transcranial direct current stimulation: evidence for homeostatic plasticity in the human motor cortex. J Neurosci 2004, 24(13):3379-3385.

78. Snodgrass JG, Corwin J: Pragmatics of measuring recognition memory: applications to dementia and amnesia. J Exp Psychol Gen 1988, 117(1):34-50.

doi:10.1186/1471-2202-12-72

Cite this article as: Hammer et al.: Errorless and errorful learning modulated by transcranial direct current stimulation. BMC Neuroscience 2011 12:72.

Submit your next manuscript to BioMed Central and take full advantage of:

- Convenient online submission

- Thorough peer review

- No space constraints or color figure charges

- Immediate publication on acceptance

- Inclusion in PubMed, CAS, Scopus and Google Scholar

- Research which is freely available for redistribution

Submit your manuscript at www.biomedcentral.com/submit
() Biomed Central 